\title{
Introduction to mammalian genome special issue: genetics of behavior
}

\author{
Lisa M. Tarantino $\cdot$ Leonard C. Schalkwyk
}

Published online: 25 January 2014

(c) Springer Science+Business Media New York 2014

The Brain Initiative, a multi-year project in the US aimed at mapping brain activity, will begin in 2014. A similar initiative in the European Union, The Human Brain Project, has recently started, and 2014 has been designated as "The Year of the Brain" by the European Brain Council. It is obvious that worldwide, a renewed emphasis has been placed on understanding the specifics of how the brain works to produce "normal" behaviors and, by extension, how these processes can go wrong and result in psychiatric and neurodevelopmental disease. Genetics figures to play a large role in these efforts.

It has been known for centuries that behaviors are inherited. These lessons were likely learned during the domestication of animals for agricultural purposes. An understanding of the exact mechanisms by which behavior was transmitted across generations was, of course, out of reach until the discovery of DNA and a greater comprehension of the role of genes. For several decades, a great deal of effort has been expended to identify genes that influence behavior. Much of this work has been performed in mice.

Early studies utilized existing genetic reference populations such as the recombinant inbred (RI) lines to identify quantitative trait loci (QTL)—genomic regions that were

\footnotetext{
L. M. Tarantino

Department of Psychiatry, School of Medicine and Division of Pharmacotherapy and Experimental Therapeutics (DPET), Eshelman School of Pharmacy, University of North Carolina, Chapel Hill, NC 27599, USA

L. C. Schalkwyk $(\square)$

MRC Social Genetic and Developmental Psychiatry (SGDP)

Centre, Institute of Psychiatry, King's College London,

De Crespigny Park, London SE5 8AF, UK

e-mail: Leonard.Schalkwyk@kcl.ac.uk
}

associated with behavioral phenotypes. These early studies, while useful, were limited by low power due to the sample size limitations of existing experimental populations. Larger studies of intercross or backcross populations between two inbred mouse strains increased power, but reduced resolution to detect manageable QTL regions in which to effectively identify candidate genes. As a result, hundreds of QTL for behavior have been reported to date, but only a handful of genes have been identified using these approaches.

This special issue of mammalian genome on genetics of behavior comes at a time when the intersection of new genomic technologies and experimental populations in the mouse and a greater appreciation and understanding of environmental influences are accelerating the identification of genes that influence behavior. Incremental lessons learned from the successes and failures of the past have driven the development of new experimental approachesmany of which are outlined in the pages of this issue.

The use of reference populations and experimental mouse crosses to identify QTL highlighted several drawbacks: (1) the sample size of existing RI lines was too small, (2) QTL from experimental crosses were prohibitively large and (3) the genetic variance sampled in standard two-strain crosses was low. The review by Chesler describes two new experimental populations, the collaborative cross (CC) and diversity outcross (DO), that were designed to overcome these issues. Chesler (2014) provides a framework by which the CC and DO lines can be used to study the genetics of complex behavioral phenotypes and asserts that the CC and DO lines will also be useful for separating linked, but functionally unrelated co-expressed gene clusters.

The genetical genomics approach of integrating gene expression profiling with phenotypic QTL analysis is often 
used to identify relevant candidate genes. The use of gene expression as a quantitative trait to identify expression QTL (eQTL) results in the identification of eQTL peaks that overlap with behavioral QTL. Performing these analyses in genetically complex populations with more than two founders, such as the CC and the DO, and using new high-throughput technologies, such as RNA-Seq, present considerable challenges. A review by Hitzemann et al. (2014) describes analytical and technical issues that must be dealt with when designing and running these studies.

The ultimate goal of animal behavior genetic studies is translating the findings into humans with psychiatric disease. Much has been learned over the last several decades about neurodevelopment, neural circuitry, and genetics. What has become clear is that the current diagnostic criteria for many psychiatric diseases may be imposing limits on how we think about these illnesses and how we model the symptoms or endophenotypes in animals. Importantly, these limits may be constraining our ability to move forward and accurately define the overlapping pathophysiology of many psychiatric diseases. The NIMH Research Domain Criteria or RDoC, described by Simmons and Quinn (2014), was developed to provide a framework for clinical diagnoses and basic research aimed at examining underlying neurobiological mechanisms.

The ability to compare across species is crucial for determining translational significance, but the annotation of phenotypes in animal models is not easily comparable across studies and with human data. Hoehndorf et al. (2014) describe the neuro behavior ontology that was developed to produce a comprehensive annotation of animal behavior and human psychiatric disease that encompasses information from behavioral, gene expression, and knockout studies.

Behavior is of course dependent on the environment as well as genetic factors, and this special issue also covers some of the ways the field is expanding to study environment factors and their genetic interactions. Grissom et al. (2014) review the literature on effects on offspring of maternal exposures to enriched and impoverished diets as well as touching on drugs of abuse and possible intergenerational effects.

Moloney et al. (2014) give a very detailed survey of the literature on the possible effects of the gut flora both in infancy and in adult life on the CNS and behavior. A particularly valuable feature of the review is that it covers human studies in parallel with preclinical (primarily rodent) experimental work.

Ibarra-Soria et al. (2014) review the vomeronasal organ and its behavioral implications. The vomeronasal organ is either absent or rudimentary in human and involved in innate responses to chemical signaling in most mammals. The two classes of G-protein coupled receptors important in the vomeronasal organ have been difficult to study but are of enormous biological interest.

There is currently a lot of interest in epigenetics as the probable mechanism for coupling environmental exposures with gene expression. One of the most extensively studied epigenetic phenomena is genomic imprinting, which records the parent of origin of approximately 100 genomic regions. The detailed study of these has given us most of our current understanding of epigenetic mechanisms of gene regulation. Dent and Isles (2014) review in detail two genes, Nesp and Grb10, which have behavioral phenotypes in the adult, and prompt us to think about the potential role of parent of origin in human genetic studies of psychiatric phenotypes as well.

We are in a time of tremendous opportunity for genetics, with genomic and genetic data and resources developing so quickly that whole new ways of thinking open up each year. The coupling of model species and human studies is becoming tighter as more of the common biology is uncovered, and this is lending a renewed impetus to the study of behavior genetics, epigenetics, and environmental interaction. A special issue such as this cannot hope to be comprehensive, but we hope we have assembled some stimulating syntheses of current topics in the field.

\section{References}

Chesler EJ (2014) Out of the bottleneck: the Diversity Outcross and Collaborative Cross mouse populations in behavioral genetics research. Mamm Genome 25:3-11. doi: 10.1007/s00335-0139492-9

Dent CL, Isles AR (2014) Brain-expressed imprinted genes and adult behaviour: the example of Nesp and Grb10. Mamm Genome 25:87-93. doi: 10.1007/s00335-013-9472-0

Grissom N, Bowman N, Reyes TM (2014) Epigenetic programming of reward function in offspring: a role for maternal diet. Mamm Genome 25:41-48. doi: 10.1007/s00335-013-9487-6

Hitzemann R, Bottomly D, Iancu O, Buck K, Wilmot B, Mooney M, Searles R, Zheng C, Belknap J, Crabbe J, McWeeney S (2014) The genetics of gene expression in complex mouse crosses as a tool to study the molecular underpinnings of behavior traits. Mamm Genome 25:12-22. doi: 10.1007/s00335-013-9495-6

Hoehndorf R, Hancock JM, Hardy NW, Mallon A-M, Schofield PN, Gkoutos GV (2014) Analyzing gene expression data in mice with the Neuro Behavior Ontology. Mamm Genome 25:32-40. doi: $10.1007 / \mathrm{s} 00335-013-9481-\mathrm{z}$

Ibarra-Soria X, Levitin MO, Logan DW (2014) The genomic basis of vomeronasal-mediated behavior. Mamm Genome 25:75-86. doi: 10.1007/s00335-013-9463-1

Moloney RD, Desbonnet L, Clarke G, Dinan TG, Cryan JF (2014) The microbiome: stress, health and disease. Mamm Genome 25:49-74. doi: 10.1007/s00335-013-9488-5

Simmons JM, Quinn KJ (2014) The NIMH Research Domain Criteria (RDoC) Project: implications for genetics research. Mamm Genome 25:23-31. doi: 10.1007/s00335-013-9476-9 